UVX 2008 (2009) 93-99

(C) EDP Sciences, 2009

DOI: $10.1051 / \mathrm{uvx} / 2009015$

\title{
Développement de nouveaux cristaux non linéaires pour la conception de dispositifs laser solides émettant dans l'ultraviolet
}

\author{
K. Xu ${ }^{1}$, L. Gheorghe ${ }^{1}$, P. Loiseau ${ }^{1}$, G. Aka ${ }^{1}$, J. Lejay $^{1}$, D. Rytz ${ }^{2}$, P. Villeval ${ }^{3}$, \\ A. Maillard ${ }^{4}$, P. Georges ${ }^{5}$, L. Mc Donagh ${ }^{6}$ et F. Salin ${ }^{6}$ \\ ${ }^{1}$ LCMCP, UMR 7574, ENSCP, 11 rue Pierre et Marie Curie, 75005 Paris, France \\ ${ }^{2}$ FEE GmbH, Struthstr. 2, 55743 Idar-Oberstein, Allemagne \\ ${ }^{3}$ Cristal Laser, Parc d'Activité du Breuil, 32 rue Robert Schumann, 54850 Messein, France \\ ${ }^{4}$ LMOPS, UMR 7132, 2 rue E. Belin, 57070 Metz, France \\ ${ }^{5}$ LCFIO, UMR 8501, Institut d'optique - campus Polytechnique, RD 128, \\ 91127 Palaiseau, France \\ ${ }^{6}$ EOLITE Systems, 11 avenue Canteranne, 33600 Pessac, France
}

\begin{abstract}
Résumé. Une technique prometteuse pour la réalisation de dispositifs laser solides émettant dans l'ultraviolet consiste à disposer en cascade plusieurs cristaux non linéaires générant des sommes de fréquence à partir d'une émission laser fondamentale située dans le proche infra-rouge. Cependant, à ce jour, peu de cristaux non linéaires offrent la possibilité de générer de manière efficace des longueurs d'onde voisines ou inférieures à $250 \mathrm{~nm}$. Dans ce but, les plus gros efforts de recherche actuels s'orientent vers des cristaux contenant des groupements borate. Après une description des principaux cristaux non linéaires proposés pour ce type d'application, nous présenterons les études que nous menons sur des matériaux de type fluoroborate, dérivés des oxoborates de terre rare et de calcium $\operatorname{TrCa}_{4} \mathrm{O}\left(\mathrm{BO}_{3}\right)_{3}(\mathrm{Tr}=$ terre rare), et sur des matériaux de structure huntite $\operatorname{TrMe}_{3}\left(\mathrm{BO}_{3}\right)_{4}(\mathrm{Me}=\mathrm{Al}, \mathrm{Ga}, \mathrm{Sc})$.
\end{abstract}

\section{INTRODUCTION}

L'intérêt pour des systèmes laser compacts émettant dans l'ultraviolet ne cesse de s'accroître du fait de leurs nombreuses applications dans les domaines de la chirurgie médicale (notamment ophtalmologique), de la microélectronique, de la photolithographie, du marquage de plastiques, de la chimie, des sciences des matériaux, ... En effet, du fait des caractéristiques du rayonnement UV (aptitude à être fortement focalisé, haute énergie permettant d'induire des ruptures de liaisons chimiques, ...), de tels lasers pourraient être utilisés en microélectronique pour le micro-usinage de circuits imprimés et pour l'inspection ou la réalisation de masques sur semi-conducteurs. Dans ce contexte, ils devraient permettre à terme de supplanter les lasers à gaz actuellement utilisés (lasers excimères $\mathrm{XeCl}, \mathrm{KrF}, \mathrm{ArF}$ voire $\mathrm{F}_{2}$ émettant respectivement à $351 \mathrm{~nm}, 248 \mathrm{~nm}, 193 \mathrm{~nm}$ et $157 \mathrm{~nm}$ ): ces derniers utilisent des gaz corrosifs dans des tubes à décharge de haute tension, sont encombrants et nécessitent une maintenance régulière et coûteuse. Au contraire, les lasers solides ont potentiellement des durées de vie plus longues, engendrent des coûts d'utilisation plus faibles et permettent d'envisager une miniaturisation des dispositifs. De plus, ils sont capables d'opérer à des taux de répétition plus élevés en régime impulsionnel, ils sont caractérisés par des largeurs spectrales d'émission plus fines et de meilleures qualités de faisceau, et ils permettent la conception de sources laser UV accordables.

Actuellement, la voie la plus explorée pour la réalisation de tels dispositifs laser solides consiste à disposer en cascade plusieurs cristaux non linéaires générant des sommes de fréquence à partir d'une émission laser fondamentale située dans le proche infra-rouge (émission fournie par un cristal dopé néodyme ou ytterbium vers $1 \mu \mathrm{m}$ par exemple). De cette façon, la longueur d'onde fondamentale d'émission laser d'un cristal $\mathrm{Y}_{3} \mathrm{Al}_{5} \mathrm{O}_{12}: \mathrm{Nd}$ (YAG : $\mathrm{Nd}$ ) à $1064 \mathrm{~nm}$ pourrait d'abord être doublée en 
fréquence $(2 \omega, \lambda=532 \mathrm{~nm})$ puis convertie en longueurs d'onde ultraviolettes par génération de $3^{\text {ème }}$, $4^{\grave{e ̀ m e}}, 5^{\text {ème }}, \ldots$ harmonique $(3 \omega, 4 \omega, 5 \omega, \ldots$ à $\lambda=355 \mathrm{~nm}, 266 \mathrm{~nm}, 213 \mathrm{~nm}, \ldots$ respectivement $)$. Dans ce but, les plus gros efforts de recherche actuels s'orientent vers des cristaux à base de borates (non centrosymétriques et non cubiques pour permettre l'obtention d'un phénomène non linéaire) du fait des bonnes propriétés générales de cette classe de matériaux en terme de transparence dans l'ultraviolet, d'efficacité non linéaire et de résistance aux dommages laser, combinés à leur grande richesse chimique [1]. Cependant, à ce jour, peu de cristaux non linéaires offrent la possibilité de générer de manière efficace des longueurs d'onde voisines ou inférieures à $250 \mathrm{~nm}$, si bien que le champ d'investigation dans le domaine des matériaux non linéaires pour l'ultraviolet reste largement ouvert. Après une revue des principaux cristaux non linéaires montrant une réelle aptitude pour la conversion de fréquence dans le domaine ultraviolet, nous présenterons les investigations que nous menons d'une part sur des matériaux de type oxoborate de terre rare et de calcium $\left(\operatorname{TrCa}_{4}\left(\mathrm{BO}_{3}\right)_{3} \mathrm{O}\right.$, Tr: terre rare) et de leurs dérivés comme $\mathrm{Ca}_{5}\left(\mathrm{BO}_{3}\right)_{3} \mathrm{~F}$, et d'autre part sur des matériaux de structure huntite comme $\mathrm{YAl}_{3}\left(\mathrm{BO}_{3}\right)_{4}$ ou $\mathrm{Y}_{x} \mathrm{La}_{y} \mathrm{Sc}_{4-x-y}\left(\mathrm{BO}_{3}\right)_{4}$.

\section{PRINCIPALES FAMILLES DE CRISTAUX BORATE POUR LA CONVERSION DE FRÉQUENCE DANS LE DOMAINE ULTRAVIOLET}

\section{1. $\beta-\mathrm{BaB}_{2} \mathrm{O}_{4}(\beta-\mathrm{BBO})$}

La forme basse température $\beta$ de $\mathrm{BaB}_{2} \mathrm{O}_{4}$, non centro-symétrique de groupe d'espace $\mathrm{R} 3 \mathrm{c}$ et stable en dessous de $925^{\circ} \mathrm{C}$ (la température de fusion est d'environ $1095^{\circ} \mathrm{C}$ ), a été découverte dans la première moitié des années 1980 [2,3] : c'est l'un des tous premiers borates anhydres à avoir été spécifiquement développé pour l'optique non linéaire. Il s'agit aujourd'hui d'un matériau commercial dont la cristallogenèse peut se faire en flux [4] ou par la méthode Czochralski sous fort gradient de température à partir de mélanges de composition stoechiométrique [5]. La structure de $\beta-\mathrm{BaB}_{2} \mathrm{O}_{4}$ est composée d'anneaux $\left[\mathrm{B}_{3} \mathrm{O}_{6}\right]$ lui conférant une biréfringence assez grande $(\Delta \mathrm{n}=0,118$ à $589 \mathrm{~nm})$ ainsi que certains coefficients non linéaires élevés $\left(\mathrm{d}_{22}(1064 \mathrm{~nm})=2,2 \mathrm{pm} . \mathrm{V}^{-1}\right)$ [6]. Pour une interaction de type I, il présente une longueur d'onde fondamentale de coupure pour le doublage de fréquence de $410 \mathrm{~nm}$ : il permet donc d'obtenir le quatrième harmonique à $266 \mathrm{~nm}$ d'un laser YAG: Nd à $1064 \mathrm{~nm}$ par simple doublage de fréquence de son second harmonique à $532 \mathrm{~nm}$. De cette façon, 2,1 W à $266 \mathrm{~nm}$ ont pu être atteints à partir de $7 \mathrm{~W}$ à $1064 \mathrm{~nm}$ (laser YAG : Nd Q-switched à $5 \mathrm{kHz}$ ) [7].

Bien que très utilisé, $\beta-\mathrm{BaB}_{2} \mathrm{O}_{4}$ présente plusieurs désavantages pour ses applications :

- relativement hygroscopique, sa mise en forme reste assez délicate

- sa large biréfringence est responsable d'un important phénomène de double réfraction ("walk-off") qui limite par exemple la longueur utile du cristal pour la conversion de fréquence

- les possibilités d'accord de phase de $\beta-\mathrm{BaB}_{2} \mathrm{O}_{4}$ dans l'UV seraient plus vastes si sa transparence dans l'ultraviolet était plus étendue (seuil de coupure à $189 \mathrm{~nm}$ )

- le seuil de dommage laser reste modeste $\left(13 \mathrm{~J} . \mathrm{cm}^{-2}\right.$ à $\left.1064 \mathrm{~nm}, 10 \mathrm{~ns}\right)$ pour pouvoir envisager des applications de forte puissance.

\section{2. $\mathrm{LiB}_{3} \mathrm{O}_{5}(\mathrm{LBO})$ et $\mathrm{CsLiB}_{6} \mathrm{O}_{10}(\mathrm{CLBO})$}

Les propriétés optiques non linéaires de $\mathrm{LiB}_{3} \mathrm{O}_{5}(\mathrm{LBO})$ ont été démontrées en 1989 par Chen et al. [8]. De groupe d'espace Pna2 ${ }_{1}$, il se décompose à $834^{\circ} \mathrm{C}$ [9] si bien que sa cristallogenèse se fait en flux. Cette dernière est aujoud'hui très bien maîtrisée et LBO est disponible commercialement en grandes dimensions (des spécimens de plus de $1 \mathrm{~kg}$ et de très bonne qualité optique peuvent être obtenus) avec une excellente qualité optique. Constitué de groupements borate de type $\left[\mathrm{B}_{3} \mathrm{O}_{7}\right]$, ce cristal présente une transparence étendue dans l'UV (seuil de coupure vers $158 \mathrm{~nm}$ ), une bonne efficacité non linéaire $\left(\mathrm{d}_{32}(1064 \mathrm{~nm})=0,85 \mathrm{pm} . \mathrm{V}^{-1}[10]\right)$, un seuil de dommage laser assez élevé $\left(25 \mathrm{~J} . \mathrm{cm}^{-2}\right.$ à 
$1064 \mathrm{~nm}, 10 \mathrm{~ns})$ et une biréfringence modérée $(\Delta \mathrm{n}=0,042$ à $589 \mathrm{~nm})$. Il est ainsi largement employé pour les générations du second ou du troisième harmonique d'un laser comme YAG : $\mathrm{Nd}$. Son domaine d'application dans l'UV est toutefois limité par sa biréfringence insuffisante pour autoriser, par exemple, des conditions d'accord de phase pour la génération du quatrième harmonique à $266 \mathrm{~nm}$ par simple doublage de fréquence : la plus courte longueur d'onde que puisse doubler en fréquence LBO est $554 \mathrm{~nm}$.

De structure parente de LBO (groupe d'espace $\mathrm{I} \overline{4} 2 \mathrm{~d}), \mathrm{CsLiB}_{6} \mathrm{O}_{10}(\mathrm{CLBO})$ a été reporté dans la littérature en 1995 [11]. Contrairement à LBO, CLBO fond de manière congruente à $848^{\circ} \mathrm{C}$ : sa cristallogenèse apparaît donc plus aisée que celle de LBO et elle peut être envisagée à partir de mélanges de composition stoechiométrique (méthodes Kyropoulos, voire Czochralski) ou le plus souvent en flux à partir de bains légèrement décalés en composition [12]. Par rapport à LBO, CLBO offre des efficacités non linéaires et une tenue au flux laser similaires. Bien que sa transparence dans l'UV soit plus restreinte (seuil de coupure de $180 \mathrm{~nm})$, il est caractérisé par une biréfringence plus élevée $(\Delta \mathrm{n}=0,052$ à $589 \mathrm{~nm})$ et ses possibilités d'accord de phase sont nettement déplacées vers les plus courtes longueurs d'onde: la limite inférieure de CLBO pour le doublage de fréquence est de $476 \mathrm{~nm}$. Ainsi, avec un cristal de CLBO de $15 \mathrm{~mm}, 23 \mathrm{~W}$ à $266 \mathrm{~nm}$ ont été obtenus à partir de $100 \mathrm{~W}$ de vert fournis par un laser YAG: $\mathrm{Nd}$ doublé en fréquence (laser Q-switched à $10 \mathrm{kHz}$ ) [12]. CLBO présente néanmoins une très forte hygroscopie qui freine son essor commercial : outre les difficultés qu'il faut surmonter pour mettre en forme ce cristal, son utilisation nécessite en général de le maintenir vers $140-160^{\circ} \mathrm{C}$ pour éviter toute détérioration dans les dispositifs laser.

\section{3. $\mathrm{KBe}_{2} \mathrm{BO}_{3} \mathrm{~F}_{2}(\mathrm{KBBF})$ et $\mathrm{Sr}_{2} \mathrm{Be}_{2} \mathrm{~B}_{2} \mathrm{O}_{7}(\mathrm{SBBO})$}

$\mathrm{KBe}_{2} \mathrm{BO}_{3} \mathrm{~F}_{2}$ (KBBF) [13] et $\mathrm{Sr}_{2} \mathrm{Be}_{2} \mathrm{~B}_{2} \mathrm{O}_{7}$ (SBBO) [14] suscitent un intérêt tout particulier du fait de leurs très bonnes propriétés non linéaires pour la génération de rayonnements UV. Avec un seuil de transmission dans l'UV de $155 \mathrm{~nm}$ et une biréfringence modérée $(\Delta \mathrm{n}=0,078$ à $589 \mathrm{~nm})$, KBBF offre des possibilités très étendues pour la conversion de fréquences dans l'UV avec une efficacité non linéaire moyenne $\left(\mathrm{d}_{11}(1064 \mathrm{~nm})=0,49 \mathrm{pm} . \mathrm{V}^{-1}\right)$ : il est sans équivalent pour la génération d'harmoniques dans le domaine VUV ("vacuum ultraviolet") $[15,16]$, et la plus courte longueur d'onde qu'il puisse doubler en fréquence est de $328 \mathrm{~nm}$ en type I, même s'il faut noter que, dans ce cas, l'efficacité de conversion de fréquence devient nulle du fait de la symétrie du cristal (groupe d'espace R32). Néanmoins, KBBF et SBBO, de structure lamellaire et de fusion non congruente, sont extrêmement difficiles à élaborer en flux alors que des voies de synthèse hydrothermale commencent à être explorées [17], leur synthèse implique la manipulation de béryllium (hautement toxique) et ils possèdent de piètres caractéristiques mécaniques. Par exemple, il n'a pas été possible jusqu'à maintenant d'obtenir des lames de KBBF orientées perpendiculairement à $\vec{c}$ d'épaisseur supérieure à $2 \mathrm{~mm}:$ leur utilisation dans des dispositifs laser a alors pu se faire par leur insertion dans des prismes de $\mathrm{CaF}_{2}$ maintenus par contact optique $[15,16]$.

C'est pourquoi toute une famille d'autres composés, en particulier d'alumino-borates, a été développée sur la base de $\mathrm{KBBF}$ et de $\mathrm{SBBO}$ : il s'agit par exemple de $\mathrm{BaAlBO}_{3} \mathrm{~F}_{2}$ (BABF), $\mathrm{BaAl}_{2} \mathrm{~B}_{2} \mathrm{O}_{7}$ (BABO) et $\mathrm{K}_{2} \mathrm{Al}_{2} \mathrm{~B}_{2} \mathrm{O}_{7}$ (KABO) [18-20]. Ils sont tous constitués de groupements anioniques [ $\mathrm{BO}_{3}$ ] comme KBBF et SBBO, mais leurs propriétés mécaniques sont sensiblement améliorées du fait d'un caractère lamellaire moins prononcé. La cristallogenèse en flux de KABO apparaît probablement comme la plus aisée, même si elle reste très délicate et que la qualité cristalline de KABO souffre encore d'une transparence optique altérée en dessous de $300 \mathrm{~nm}$, avec un seuil de coupure situé vers $190 \mathrm{~nm}$ [21]. $\mathrm{KABO}$ est caractérisé par une biréfringence et un coefficient non linéaire très légèrement inférieurs à ceux de $\operatorname{KBBF}\left(\Delta \mathrm{n}=0,070\right.$ à $\left.589 \mathrm{~nm}, \mathrm{~d}_{11}(1064 \mathrm{~nm})=0,47 \mathrm{pm} . \mathrm{V}^{-1}\right)$, et la longueur d'onde de coupure de $\mathrm{KABO}$ pour la génération de second harmonique en type I est de $451 \mathrm{~nm}$, bien que là encore le coefficient non linéaire effectif s'annule (groupe d'espace P32). Aussi, un rendement de conversion de fréquence de $532 \mathrm{~nm}$ en $266 \mathrm{~nm}$ de $13 \%$ a été mesuré [22]. Cependant, à ce jour, aucun des cristaux de 
la famille de KBBF et SBBO n'a atteint un degré de maturité suffisant, tout particulièrement en matière de cristallogenèse, pour être disponible commercialement.

\section{INVESTIGATION DES PROPRIÉTÉS OPTIQUES NON LINÉAIRES DE CRISTAUX DE LA FAMILLE DES OXOBORATES DE TERRE RARE ET DE CALCIUM}

\subsection{Développement de cristaux de type $\mathrm{Gd}_{1-x} \operatorname{Tr}_{x} \mathrm{Ca}_{4}\left(\mathrm{BO}_{3}\right)_{3} \mathrm{O}(\mathrm{Tr}=\mathrm{Lu}, \mathrm{Sc})$ pour un accord de phase non critique vers $800 \mathrm{~nm}$}

Les propriétés non linéaires des oxoborates de terre rare et de calcium ont pour la première fois été démontrées dans la littérature en 1996 pour $\mathrm{GdCa}_{4}\left(\mathrm{BO}_{3}\right)_{3} \mathrm{O}(\mathrm{GdCOB})$ [23] et en 1997 pour $\mathrm{YCa}_{4}\left(\mathrm{BO}_{3}\right)_{3} \mathrm{O}$ (YCOB) [24]. Cristallisant dans le groupe d'espace $\mathrm{Cm}$, ces matériaux ont le très gros avantage de pouvoir être élaborés par la technique Czochralski du fait de leur fusion (quasi-) congruente [25] $\left(1480^{\circ} \mathrm{C}\right.$ et $1510^{\circ} \mathrm{C}$ pour $\mathrm{GdCOB}$ et YCOB respectivement) : ces cristaux peuvent donc rapidement être obtenus (vitesse de cristallisation d'environ $1 \mathrm{~mm} . \mathrm{h}^{-1}$ ) en grande dimension avec une bonne qualité optique [26]. Les groupements $\left[\mathrm{BO}_{3}\right]$ de ces matériaux leur confèrent une assez bonne efficacité non linéaire $\left(\mathrm{d}_{13}(1064 \mathrm{~nm}) \approx 0,59 \mathrm{pm} . \mathrm{V}^{-1}\right)$ [27]. Par rapport à YCOB, GdCOB présente une transmission limitée dans l'UV du fait de bandes d'absorption du gadolinium en dessous de $320 \mathrm{~nm}$, et une biréfringence un peu plus faible ( $\Delta \mathrm{n}=0,035$ pour $\mathrm{GdCOB}$ et $\Delta \mathrm{n}=0,043$ pour YCOB à $589 \mathrm{~nm}$ ). Les plus courtes longueurs d'onde fondamentales que puissent doubler GdCOB et YCOB en type I sont ainsi respectivement de 824 et $723 \mathrm{~nm}$ (propagation selon Y), et YCOB est le seul à autoriser la génération de troisième harmonique (en type I uniquement) pour des longueurs d'onde laser proche IR usuelles (supérieures toutefois à $1030 \mathrm{~nm}$ ). C'est notamment pour cette dernière raison que YCOB a été retenu dans le cadre du projet américain Mercury [26] comme matériau optique non linéaire.

Même si les possibilités de conversion de fréquence dans l'UV des oxoborates de terre rare et de calcium sont assez limitées, ces matériaux ont le grand avantage de constituer une famille homogène de composés où il est permis de changer, totalement ou partiellement, la nature de la terre rare. Partant du constat que les courbes d'angles d'accord de phase se déplacent vers les plus courtes longueurs d'onde à mesure que le taille de la terre rare diminue, il est alors possible d'ajuster la composition de ces matériaux pour offrir un accord de phase à des longueurs d'onde choisies selon les directions simples de propagation que constituent les axes diélectriques : ces directions, faciles à orienter, conduisent à des conditions d'accord de phase non critique pour lesquelles l'angle de double réfraction est nul. Le succès de cette approche a tout d'abord été démontré dans le cas de la solution solide $\mathrm{Gd}_{1-x} \mathrm{Y}_{x} \mathrm{Ca}_{4}\left(\mathrm{BO}_{3}\right)_{3} \mathrm{O}$ (GdYCOB) pour le doublage (type II) et le triplage (type I) en fréquence de $1064 \mathrm{~nm}$ [28]. Nous avons chercher à étendre cette possibilité au doublage en fréquence de longueurs d'onde situées autour de $800 \mathrm{~nm}$ pouvant émaner de lasers saphir : Ti ou de diodes laser AlGaAs. Pour cela, une substitution partielle de l'ion $\mathrm{Gd}^{3+}$ (rayon ionique $94 \mathrm{pm}$ en coordinence 6 [29]) par un cation plus petit que $\mathrm{Y}^{3+}$ (rayon ionique $90 \mathrm{pm}$ ) a été envisagée pour obtenir une variation rapide de la biréfringence : les ions $\mathrm{Lu}^{3+}$ (rayon ionique $86 \mathrm{pm}$ ) et $\mathrm{Sc}^{3+}$ (rayon ionique $75 \mathrm{pm}$ ) ont ainsi été choisis. Des cristaux de compositions $\mathrm{Gd}_{1-x} \mathrm{Lu}_{x} \mathrm{Ca}_{4}\left(\mathrm{BO}_{3}\right)_{3} \mathrm{O}$ avec $\mathrm{x}=0,07$ et $\mathrm{x}=0,13$, et $\mathrm{Gd}_{1-x} \mathrm{Sc}_{x} \mathrm{Ca}_{4}\left(\mathrm{BO}_{3}\right)_{3} \mathrm{O}$ avec $\mathrm{x}=0,04$ ont été élaborés par la technique Czochralski [30], puis il a été montré qu'ils permettaient d'obtenir de bonnes performances en doublage intra-cavité d'un laser saphir : Ti continu autour de $800 \mathrm{~nm}$ [31] : un maximum de $115 \mathrm{~mW}$ a été obtenu à $407 \mathrm{~nm}$ pour 5,5 $\mathrm{W}$ de pompe (laser Verdi V5) avec un cristal de $\mathrm{Gd}_{0,96} \mathrm{Sc}_{0,04} \mathrm{Ca}_{4}\left(\mathrm{BO}_{3}\right)_{3} \mathrm{O}$, long de $6,5 \mathrm{~mm}$.

\subsection{Des oxoborates de terre rare et de calcium $\operatorname{TrCa}_{4}\left(\mathrm{BO}_{3}\right)_{3} \mathrm{O}$ au fluoroborate de calcium $\mathrm{Ca}_{5}\left(\mathrm{BO}_{3}\right)_{3} \mathrm{~F}(\mathrm{CBF})$}

Le domaine de transparence restreint des oxoborates de terre rare et de calcium (seuil de coupure de $205 \mathrm{~nm}$ pour YCOB) combiné à leur biréfringence trop modeste ne permettent pas à ces cristaux d'offir 
un vaste champ d'application dans le domaine UV. Pour remédier à ce problème, nous avons envisagé de modifier assez profondément la chimie de ces matériaux en procédant au schéma de substitution suivant: $\mathrm{Tr}^{3+}+\mathrm{O}^{2-} \rightarrow \mathrm{Ca}^{2+}+\mathrm{F}^{-}$. Du fait de l'électronégativité plus grande du fluor par rapport à l'oxygène, une transparence plus étendue dans l'UV est attendue pour le fluoroborate de calcium $\mathrm{Ca}_{5}\left(\mathrm{BO}_{3}\right)_{3} \mathrm{~F}(\mathrm{CBF})$ qui résulte de cette substitution couplée. $\mathrm{CBF}$ est isostructural des oxoborates de terre rare et de calcium [32], mais il présente une fusion non congruente : il se décompose vers $1250^{\circ} \mathrm{C}$ et sa cristallogenèse peut se faire en flux de $\operatorname{LiF}[33,34]$. Des cristaux de taille et de qualité optique suffisantes ont pu être obtenus pour évaluer les potentialités de ce matériau : avec un seuil de transmission dans l'UV situé vers 190-195 nm et une biréfringence sensiblement plus élevée que les oxoborates de terre rare et de calcium $(\Delta \mathrm{n}=0,053$ à $589 \mathrm{~nm})$, il présente des courbes d'accord de phase déplacées vers les plus courtes longueurs d'onde. Ainsi, il autorise la génération de troisième harmonique en type II pour des longueurs d'onde laser proche IR usuelles (supérieures toutefois à $989 \mathrm{~nm}$ ) contrairement à YCOB, et la plus courte longueur d'onde que CBF puisse doubler en fréquence en type I est de $577 \mathrm{~nm}$ : celle-ci reste cependant trop grande pour permettre la génération de quatrième harmonique par simple doublage de fréquence (elle ne peut se faire que par somme de fréquence $\omega+3 \omega$ ). Des études sont actuellement en cours pour notamment compléter la caractérisation des propriétés non linéaires de $\mathrm{CBF}$ : détermination des coefficients non linéaires $\mathrm{d}_{i j}\left(\left(\mathrm{~d}_{13}(1064 \mathrm{~nm}) \approx 0,39 \mathrm{pm} . \mathrm{V}^{-1}\right)\right.$ et réalisation de tests laser.

\section{INTÉRÊT DES MATÉRIAUX DE STRUCTURE HUNTITE POUR LA CONVERSION DE FRÉQUENCE DANS LE DOMAINE ULTRAVIOLET}

\section{1. $\mathrm{YAl}_{3}\left(\mathrm{BO}_{3}\right)_{4}(\mathrm{YAB})$}

La huntite, minéral de formule chimique $\mathrm{CaMg}_{3}\left(\mathrm{CO}_{3}\right)_{4}$ et de groupe d'espace $\mathrm{R} 32$, est le prototype structural de toute une variété de matériaux de composition $\operatorname{TrMe}_{3}\left(\mathrm{BO}_{3}\right)_{4}(\mathrm{Tr}=$ terre rare, $\mathrm{Me}=\mathrm{Al}, \mathrm{Ga}$, Sc) $[35,36]$ constitués de groupements $\left[\mathrm{BO}_{3}\right]$. Le membre le plus connu de cette famille est $\mathrm{YAl}_{3}\left(\mathrm{BO}_{3}\right)_{4}$ (YAB) : il présente la plus grande efficacité non linéaire $\left(\mathrm{d}_{11}(1064 \mathrm{~nm})=1,5 \pm 0,1 \mathrm{pm} . \mathrm{V}^{-1}\right)$ et un seuil de dommage laser élevé. Cependant, le YAB a essentiellement été étudié dopé par des ions comme $\mathrm{Nd}^{3+}$ ou $\mathrm{Yb}^{3+}$ en tant que cristal laser autodoubleur de fréquence [37] : il conduit à d'excellentes performances pour cette application. Alors qu'aucune caractérisation complète du YAB blanc n'ait encore encore été publiée, ce matériau suscite un regain d'intérêt comme simple cristal optique non linéaire du fait de sa biréfringence qui doit autoriser des accords de phase dans l'UV $(\Delta \mathrm{n}=0,074 \pm 0,001$ à $589 \mathrm{~nm})$, notamment pour le doublage de fréquence en type I de longueurs d'onde supérieures à $500 \mathrm{~nm}$, comme $532 \mathrm{~nm}$. Le principal frein au développement de telles applications tient à la cristallogenèse de ce matériau qui ne permet pas encore d'obtenir des cristaux de qualité optique suffisante. Le YAB se décompose vers $1200^{\circ} \mathrm{C}$ et sa cristallogenèse doit se faire en flux : les flux qui ont conduit aux meilleurs résultats à ce jour sont à base de $\mathrm{K}_{2} \mathrm{Mo}_{3} \mathrm{O}_{10}$, mais ils ne permettent pas encore de conserver une transparence optimale du cristal en dessous de $340 \mathrm{~nm}$, alors que le seuil de coupure est a priori attendu en dessous de $200 \mathrm{~nm}$. Des études sont donc en cours pour optimiser les conditions de croissance et améliorer la qualité optique de ces cristaux, en minimisant notamment l'incorporation d'impuretés délétères, sur la base de flux comme $\mathrm{K}_{2} \mathrm{Mo}_{3} \mathrm{O}_{10}$ ou d'autres flux borate alternatifs exempts d'éléments pouvant conduire à des absorptions dans l'UV.

\section{2. $\mathrm{Y}_{x} \mathrm{La}_{y} \mathrm{Sc}_{4-x-y}\left(\mathrm{BO}_{3}\right)_{4}(\mathrm{YLSB})$}

Un moyen de contourner la difficulté d'obtenir des cristaux de YAB transparents dans l'UV consiste à s'intéresser aux matériaux de formule type $\operatorname{TrSc}_{3}\left(\mathrm{BO}_{3}\right)_{4}$, non congruents, mais dont la cristallogenèse n'utilise pas $\mathrm{K}_{2} \mathrm{Mo}_{3} \mathrm{O}_{10}$ comme flux. Néanmoins, les composés stoechiométriques $\operatorname{TrSc}_{3}\left(\mathrm{BO}_{3}\right)_{4}$ n'existent que pour les plus gros lanthanides $(\mathrm{Tr}=\mathrm{La}$ à $\mathrm{Sm})$, et ils cristallisent sous différentes formes allotropiques en fonction du rapport de taille $\mathrm{r}(\mathrm{Tr}) / \mathrm{r}(\mathrm{Sc})$. Pour les plus gros lanthanides, comme $\mathrm{Tr}=\mathrm{La}$, 
$\mathrm{TrSc}_{3}\left(\mathrm{BO}_{3}\right)_{4}$ est centrosymétrique (groupe d'epace $\mathrm{C} 2 / \mathrm{c}$ ) et donc linéaire, alors que pour $\mathrm{Tr}=\mathrm{Nd}$ par exemple $\operatorname{TrSc}_{3}\left(\mathrm{BO}_{3}\right)_{4}$ est isostructural de la huntite (groupe d'espace R32) et donc non linéaire. Le matériau de base de cette famille qui s'impose pour des applications optiques dans l'UV est $\mathrm{LaSc}_{3}\left(\mathrm{BO}_{3}\right)_{4}(\mathrm{LSB})$ car il est le seul à garantir une bonne transparence dans l'UV. Cependant, comme ce matériau est centrosymétrique, il est nécessaire d'envisager une substitution partielle du lanthane par une terre rare plus petite inactive optiquement $(\mathrm{Tr}=\mathrm{Y}, \mathrm{Lu})$ pour stabiliser la forme huntite non centrosymétrique. Comme cette terre rare plus petite possède une taille intermédiaire entre le lanthane et le scandium, elle peut aussi se substituer partiellement au scandium, ce qui revient à explorer des compositions de type $\operatorname{Tr}_{x} \mathrm{La}_{y} \mathrm{Sc}_{4-x-y}\left(\mathrm{BO}_{3}\right)_{4}(\mathrm{Tr}=\mathrm{Y}, \mathrm{Lu})[38,39]$. La cristallogenèse de ces matériaux, plus aisée que celle du YAB, se fait en flux à base de $\mathrm{Li}_{6} \mathrm{~B}_{4} \mathrm{O}_{9}$ : nous avons ainsi pu obtenir des cristaux de $\mathrm{Y}_{x} \mathrm{La}_{y} \mathrm{Sc}_{4-x-y}\left(\mathrm{BO}_{3}\right)_{4}$ (YLSB) de plusieurs centimètres, avec une bonne qualité optique et un seuil de coupure dans l'UV d'environ $190 \mathrm{~nm}$, dont la caractérisation plus complète est en cours (calcul des courbes d'angle d'accord de phase, mesures d'efficacité non linéaire, tests laser).

\section{CONCLUSION}

Depuis les années 1980, les cristaux borate sont l'objet de nombreuses recherches pour des applications optiques non linéaires dans le domaine du proche-IR, visible et UV, mais ce sont aujourd'hui surtout les applications dans le domaine UV qui mobilisent le plus de travaux. Dans ce cas, les critères de choix des matériaux borate deviennent très sélectifs : ils doivent notamment offrir une transparence étendue dans l'UV, une biréfringence suffisante $(\Delta \mathrm{n} \sim 0,07)$, une bonne tenue au flux laser et, si possible, ne pas être hygroscopique. Ainsi, dans le cas de la génération du quatrième harmonique d'un laser YAG : Nd par simple doublage de fréquence de $532 \mathrm{~nm}$, seuls deux cristaux borate sont disponibles commercialement : $\beta$-BBO, limité en performances par son angle de double réfraction élevé et sa tenue moyenne au flux laser, et CLBO, extrêmement hygroscopique. Le verrou essentiel au développement de nouveaux cristaux est leur cristallogenèse : c'est le cas des matériaux de la famille de KBBF et SBBO. Nous avons ainsi entrepris des investigations sur des cristaux non hygroscopiques comme CBF et YLSB qui devraient dans un premier temps ouvrir des perspectives très intéressantes pour le triplage de fréquence dans l'UV vers $355 \mathrm{~nm}$. Enfin, nous cherchons également à optimiser la cristallogenèse du YAB afin d'améliorer sa transparence dans l'UV : il s'agit actuellement du principal obstacle à son utilisation dans des dispositifs laser de génération de troisième et quatrième harmonique.

\section{Remerciements}

L'ensemble des études présentées ci-dessus bénéficie d'un soutien financier de la part de l'ANR (Agence Nationale de la Recherche), dans le cadre d'un projet ANR-blanc nommé "UV-borates", sous la référence ANR-06-BLAN0169-01.

Nous remercions aussi M. Jacquet et M. Escudier de l'atelier d'optique et de rayons X de l'Institut des NanoSciences de Paris pour leur contribution à l'orientation et au polissage des cristaux.

\section{Références}

[1] P. Becker, Adv. Mater. 10, (1998) 979.

[2] J. Liebertz, S. Stähr, Z. Kristallogr. 165, (1983) 91.

[3] C. Chen, B. Wu, A. Jiang, G. You, Sci. Sin. B 28, (1985) 235.

[4] M. Roth, D. Perlov, J. Cryst. Growth 169, (1996) 734.

[5] H. Kouta, Y. Kuwano, J. Cryst. Growth 114, (1991) 676.

[6] I. Shoji, H. Nakamura, K. Ohdaira, T. Kondo, R. Ito, T. Okamoto, K. Tatsuki, S. Kubota, J. Opt. Soc. Am. B 16(4), (1999) 620.

[7] L. B. Chang, S. C. Wang, A. H. Kung, Opt. Commun. 209, (2002) 397. 
[8] C. Chen, Y. Wu, A. Jiang, B. Wu, G. You, R. Li, S. Lin, J. Opt. Soc. Am. B 6(4), (1989) 616.

[9] B. S. R. Sastry, F. A. Hummel, J. Am. Ceram. Soc. 42(5), (1959) 216.

[10] D. A. Roberts, IEEE J. Quant. Electr. 28(10), (1992) 2057.

[11] Y. Mori, I. Kuroda, S. Nakajima, T. Sasaki, S. Nakai, J. Cryst. Growth 156(3), (1995) 307.

[12] T. Sasaki, Y. Mori, M. Yoshimura, Opt. Mater. 23, (2003) 343.

[13] L. Mei, Y. Wang, C. Chen, B. Wu, J. Appl. Phys. 74, (1993) 7014.

[14] C. Chen, Y. Wang, B. Wu, K. Wu, W. Zeng, L. Yu, Nature 322, (1995) 373.

[15] C. Chen, Opt. Mater. 26, (2004) 425.

[16] G. Wang, X. Wang, Y. Zhou, Y. Chen, C. Li, Y. Zhu, Z. Xu, C. Chen, Appl. Phys. B 91, (2008) 95.

[17] C. D. McMillen, J. W. Kolis, J. Cryst. Growth 310, (2008) 2033.

[18] Z.-G. Hu, M. Yoshimura, Y. Mori, T. Sasaki, J. Cryst. Growth 275, (2005) 232.

[19] C. Chen, Z. Lin, Z. Wang, Appl. Phys. B 80, (2005) 1.

[20] C. T. Chen, L. Bai, Z. Z. Wang, R. K. Li, J. Cryst. Growth 292, (2006) 169.

[21] L. Liu, C. Chen, J. Cryst. Growth 292, (2006) 472.

[22] J. H. LU, G. L. Wang, Z. Y. Xu, C. T. Chen, J. Y. Wang, C. Q. Zhang, Y. G. Liu, Chin. Phys. Lett. 19(5), (2002) 680.

[23] G. Aka, A. Kahn-Harari, D. Vivien, J.-M. Benitez, F. Salin, J. Godard, Eur. J. Solid State Inorg. Chem. 33, (1996) 727.

[24] T. Iwai, M. Kobayashi, H. Furuya, Y. Mori, T. Sasaki, Jpn. J. Appl. Phys. 36, (1997) L276.

[25] D. Klimm, S. Ganschow, R. Bertram, J. Doerschel, V. Bermudez, A. Klos, Mater. Res. Bull. 37(10), (2002) 1737.

[26] Y. Fei, B. H. T. Chai, C. A. Ebbers, Z. M. Liao, K. I. Schaffers, P. Thelin, J. Cryst. Growth 290, (2006) 301.

[27] M. V. Pack, D. J. Amstrong, A. V. Smith, J. Opt. Soc. Am. B 22(2), (2005) 417.

[28] T. Sasaki, Y. Mori, M. Yoshimura, Y. K. Yap, T. Kamimura, Mater. Sci. Eng. R 30, (2000) 1.

[29] R. D. Shannon, Acta Crystallogr. A 32, (1976) 751.

[30] L. Gheorghe, P. Loiseau, G. Aka, V. Lupei, J. Cryst. Growth 294(2), (2006) 442.

[31] M. T. Andersen, J. L. Mortensen, S. Germershausen, P. Tidemand-Lichtenberg, P. Buchhave, L. Gheorghe, V. Lupei, P. Loiseau, G. Aka, Optics Express 15(8), (2007) 4893.

[32] S. Lei, Q. Huang, Y. Zheng, A. Jiang, C. Chen, Acta Crystallogr. C 45, (1989) 1861.

[33] G. Chen, Y. Wu, P. Fu, J. Cryst. Growth 292, (2006) 449.

[34] K. Xu, P. Loiseau, G. Aka, R. Maillard, A. Maillard, T. Taira, Optics Express 16(22), (2008) 17735.

[35] N. I. Leonyuk, L. I. Leonyuk, Prog. Crystal Growth and Charact. 31, (1995) 179.

[36] N. I. Leonyuk, Prog. Crystal Growth and Charact. 31, (1995) 279.

[37] A. Brenier, D. Jaque, A. Majchrowski, Opt. Mater. 28, (2006) 310.

[38] N. Ye, J. L. Stone-Sundberg, M. A. Hruschka, G. Aka, W. Kong, D. A. Keszler, Chem. Mater. 17, (2005) 2687.

[39] W. Li, L. Huang, G. Zhang, N. Ye, J. Cryst. Growth 307, (2007) 405. 